\title{
Superconductivity and antiferromagnetism as interfering orders in organic conductors
}

\author{
C. Bourbonnais ${ }^{1,2}$ and A. Sedeki ${ }^{1}$ \\ ${ }^{1}$ Regroupement Québécois sur les Matériaux de Pointe, Département de Physique, RQMP, Université de Sherbrooke, Sherbrooke, Québec, \\ Canada, $J 1 K-2 R 1$ \\ ${ }^{2}$ Canadian Institute of Advanced Research, Toronto, Canada.
}

\begin{abstract}
Superconductivity in the Bechgaard salts series of quasi-one-dimensional organic conductors occurs on the verge of spin-density-wave ordering when hydrostatic pressure is applied. The sequence of instabilities is intimately connected to normal state anomalies in various quantities like the temperature dependence of electrical transport and nuclear spin-lattice relaxation rate. We discuss how such a connection takes its origin in the interference between the different pairing mechanisms responsible for antiferromagnetism and superconductivity, a duo that can be comprehended in terms of a weak coupling renormalization group theory. The recent developments along this line of though are presented in relation to experiments.
\end{abstract}

Keywords: Organic conductors, superconductivity, antiferromagnetism, scaling theory

\section{Introduction}

Superconductivity in organic conductors was first observed more than three decades ago in the quasi-one-dimensional metal (TMTSF) ${ }_{2} \mathrm{PF}_{6}$ (bi-tetramethyltetraselenafulvalene hexafluorophosphate), a member of the famous Bechgaard salt series, (TMTSF $)_{2} X\left(X=\mathrm{PF}_{6}, \mathrm{AsF}_{6}, \ldots\right)$ [1]. Manifestation of superconductivity (SC) in this series emerges under pressure close by a magnetic instability of the metallic state against the formation of spin-density-wave (SDW) order [2, 3]. This sequence of phases along with its share of anomalies in the normal state, soon appeared rather unfamiliar and at odds with what was found until then in classical superconductors. The closeness of antiferromagnetism and superconductivity was not unique to the Bechgaard salts, but was also present in different classes of materials among which the little older 'heavy fermions' materials [4, 5], followed later on by the high- $T_{c}$ cuprates [6, 7, 8], layered organics [9, 10, 11, 12, 13], and more recently by iron-based superconductors [14, 15].

In spite of the considerable progress achieved in the understanding of correlated electron systems in the last decades, no consensus is yet to be found about the role played by antiferromagnetism for Cooper pairing in each of the above categories of materials, as well as about the possibility to extract some common view that would link pairing with magnetism across them. In face of these difficulties, the study of low- $T_{c}$ superconductors like the Bechgaard salts may represent a simpler avenue of investigation, providing key insight into this enduring issue. Experiments in these molecular compounds are generally compatible with the pronounced itinerant character of carriers and the existence of a well-defined quasi-one-dimensional - open - Fermi surface. This provides substantial grounds for a relatively simple weak coupling formulation of the problem of competing instabilities taking place in these anisotropic electron systems. Its best formulation is given in terms of the quasi-1D electron gas model whose consequences can be worked out fairly well by the renormalization group (RG) method [16]. RG proved to be a successful strategy for taking into account the contribution of both particle-hole and particle-particle scatterings responsible for SDW and SC pairings in the vicinity of the Fermi surface. Appearing simultaneously in perturbation theory, these scattering processes interfere with one another at arbitrary order, which can lead for instance to their mutual reinforcement. The consequences of this mixing are far from being trivial. Under certain conditions related to nesting deteriorations in the particle-hole channel, resulting for example from the application of pressure, interference acts as the mechanism of onset of magnetic pairing for d-wave superconductivity from an inhibited SDW instability. The SDW-SCd sequence 
of transitions thus obtained versus nesting alterations has been found to capture the essentials of the phase diagram of the Bechgaard salts under pressure.

The impact of interference between primary scattering channels is not restricted to the transition lines of the phase diagram, but is found to affect the properties of the normal phase as well. It is where it can supply a consistent explanation for the observation of a linear- $T$ resistivity [17, 18], and a Curie-Weiss nuclear relaxation rate [19, 20], whose amplitudes show some amazing correlation with the superconducting instability line of the phase diagram under pressure.

The purpose of this capsule review is to discuss some recent experimental results about the phase diagram of the prototype organic superconductor (TMTSF) ${ }_{2} \mathrm{PF}_{6}$, alongside electrical resistivity and NMR relaxation rate anomalies of its metallic state. We then summarize the latest theoretical developments of the renormalisation group method in the framework of the quasi-one-dimensional electron gas model, providing the logical link needed to understand these key features displayed by quasi-one-dimensional organic superconductors.

\section{Bechgaard salts: some background of experimental results}

Although early band calculations predicted that these systems should be metallic, the first ambient pressure resistivity measurements on the prototype compounds of the series, (TMTSF) ${ }_{2} \mathrm{PF}_{6}$ and $(\mathrm{TMTSF})_{2} \mathrm{AsF}_{6}$, revealed instead the existence of a metal-insulator transition at $T_{\mathrm{SDW}} \sim 12 \mathrm{~K}[21]$. Contrary to the situation encountered in organic materials that preceded the discovery of the Bechgaard salts, like the celebrated compound TTF-TCNQ [2], the low pressure state was not the result of a charge-density-wave or a Peierls instability, but was rather magnetic in character caused by the onset of SDW order. The fingerprints of long-range SDW correlations at the approach of $T_{\mathrm{SDW}}$ have been evidenced by several techniques. The Nuclear Magnetic Resonance (NMR) probe is one of them. At the approach of $T_{\mathrm{SDW}}$, critical SDW fluctuations give rise to a singular growth of staggered local magnetic field resulting in a square-root singularity of the NMR spin-lattice relaxation rate, $T_{1}^{-1}$, as shown by the ${ }^{77} \mathrm{Se}$ NMR data of Figure 2-b for $(\mathrm{TMTSF})_{2} \mathrm{PF}_{6}[20,22]$. Furthermore, from the analysis of the proton $\left({ }^{1} \mathrm{H}\right) \mathrm{NMR}$ line shape in the SDW ordered state of (TMTSF) ${ }_{2} \mathrm{PF}_{6}$, it was also possible to simulate the spatial distribution of the dipolar field and then extract the SDW modulation wave vector $\mathbf{q}_{0}$ [23]. The value $\mathbf{q}_{0}=\left(2 k_{F}, \frac{1}{4} b^{*}\right)$ thus obtained for the staggered magnetization in the $a b$ plane coincides, within experimental accuracy, with the best nesting vector of the Fermi surface found by band calculations [24]. This correspondence is of importance since it shows that the SDW transition in (TMTSF) ${ }_{2} X$ is an instability of the electron system driven, besides repulsive interactions, by nesting of the Fermi surface at the same $\mathbf{q}_{0}$, an important factor in favor of a weak coupling description of the SDW instability in these materials.

Under hydrostatic pressure, $T_{\mathrm{SDW}}$ decreases smoothly and ultimately undergoes a rapid decline near a pressure threshold of about $8 \mathrm{kbar}$ for (TMTSF) ${ }_{2} \mathrm{PF}_{6}$. There, the resistivity first shows an insulating SDW behavior and then drops to zero following the onset of superconducting order at the temperature $T_{c}$ (Fig. 1. - a). Reentrant superconductivity within the SDW phase is apparent over an interval of more or less one kbar in which SC coexists in a segregated form with SDW [25, 26]. By increasing pressure up to some critical value $P_{c}, T_{c}$ reaches a maximum $(\sim 1.2 \mathrm{~K})$ that defines the SDW-SC juncture where $T_{\mathrm{SDW}} \approx T_{c}$. Above $P_{c}$, reentrance gives way to a simple metal to SC transition characterized by a rapid downturn of $T_{c}$ toward very small values beyond $20 \mathrm{kbar}$ (Fig. 1-a). The nature of the superconducting order parameter in (TMTSF) $)_{2} X$, either singlet or triplet, along with the presence of nodes or not on the Fermi surface, have been much debated. However, the current experimental status appears to tip the scale in favor of a singlet order parameter with nodes [27, 28, 29].

A striking feature of the normal phase resistivity, close to $P_{c}$, is shown by its temperature dependence: the longitudinal resistivity $\rho_{a}$ accurately fits the non Fermi liquid linear expression, $\rho_{a}(T)=\rho_{0, a}+A T$, where $\rho_{0, a}$ is the residual resistivity and $A>0[17,18]$. In (TMTSF) ${ }_{2} \mathrm{PF}_{6}$ at $11.8 \mathrm{kbar}$ for example, linear- $T$ resistivity is detected from $8 \mathrm{~K}$ or so down to $T_{c}$; it even extends toward much lower temperature when a small magnetic field is applied to suppress $T_{c}$. Away from $P_{c}, \rho_{a} v s T$ modifies in a distinctive way. Resistivity ceases to be exclusively linear and acquires some curvature that satisfactorily fits the polynomial form $\rho_{a}(T)=\rho_{0, a}+A T+B T^{2}$. This can be interpreted as a Fermi liquid component that sets in under pressure, becoming in turn the main contribution to resistivity at very high pressure, namely where the linear- $T$ resistivity component, like $T_{c}$, becomes vanishingly small (Figs. 1 - b and 2-a). This notable correlation between $A$ and the strength of superconductivity shows that inelastic scattering and Cooper pairing are interrelated. This feature turns out to be not unique to the Bechgaard salts [17, 18, 7], but is also shared by other 

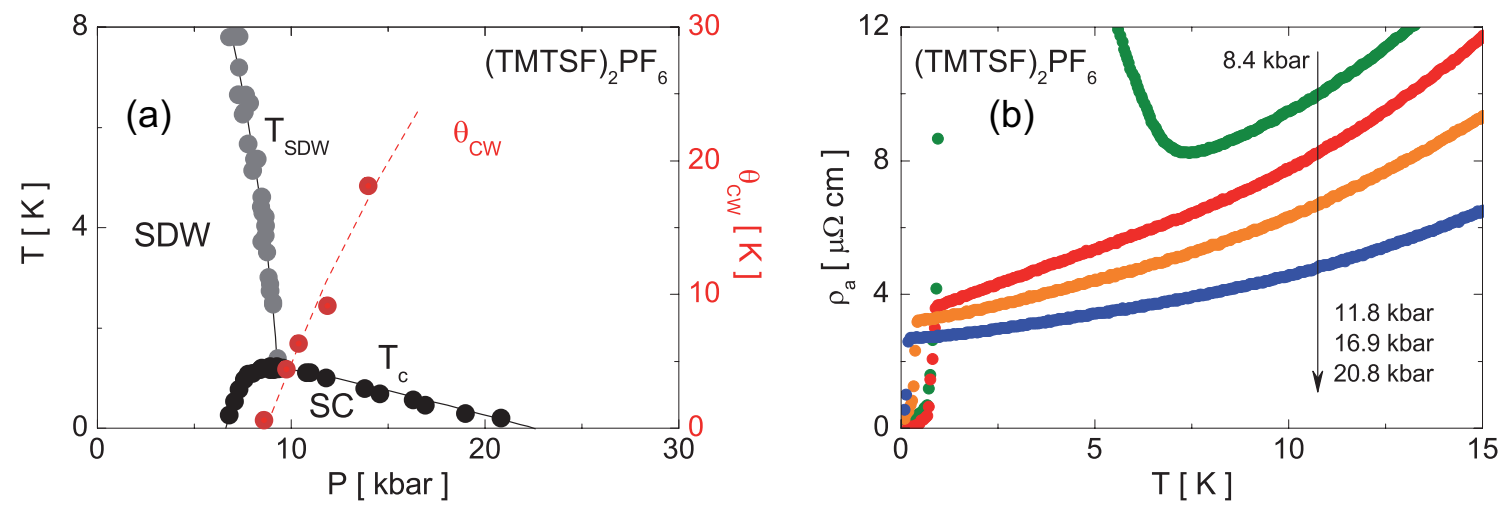

Figure 1: a) Pressure-Temperature phase diagram of (TMTSF) ${ }_{2} \mathrm{PF}_{6}$ (after Ref. [17]); also shown the variation of the Curie-Weiss scale $\Theta$ under pressure extracted from the NMR data of Fig. 2-b [20] (owing to a different low temperature determination of pressure in these two sets of experiments, a rigid, positive, shift of $3 \mathrm{kbar}$ has been applied to the pressure scale of Fig. 2fb); b) Longitudinal resistivity $v s$ temperature for $(\mathrm{TMTSF})_{2} \mathrm{PF}_{6}$ at different pressures.

categories of unconventional superconductors showing close proximity with antiferromagnetic ordering, especially in the pnictides [17, 30] and high-Tc cuprates [31, 32, 7, 33].

Connections between superconductivity and deviations from the Fermi liquid predictions in the same region of the normal state are not restricted to electrical transport, but are also found in other quantities. The NMR spin-lattice relaxation rate $T_{1}^{-1}$ is one of these [20, 22]. If one first looks at the magnetic sector of the phase diagram of Figure 1 . a, $T_{1}^{-1}$ of Figure 2 -b displays the characteristic square root singularity $\sim\left(T-T_{\mathrm{SDW}}\right)^{-1 / 2}$, which confirms the onset of long-range three-dimensional SDW correlations at the approach of $T_{\mathrm{SDW}}[22,34]$. In the superconducting sector above $P_{c}$, the critical behavior is suppressed, but an anomalously large enhancement of $T_{1}^{-1}$ remains (Figure 2 -b); its amplitude is huge close to $P_{c}$ and reduces progressively as pressure is raised. The origin of the anomaly lies in the presence of short-range SDW spin fluctuations whose amplitude shows persistent growing down to $T_{c}$ [35, 16], that is in the same temperature region where linear- $T$ resistivity is seen (Fig. 1 - b). The temperature profile of relaxation rate differs there from the Korringa law, $\left(T_{1} T\right)^{-1} \sim$ const., expected for a Fermi liquid; it rather exhibits an enhancement following the Curie-Weiss behavior $\left(T_{1} T\right)^{-1} \sim(T+\Theta)^{-1}$ [19]. The Curie-Weiss scale, $\Theta$, linked to the amplitude of the anomaly and in a way to the characteristic energy scale of spin fluctuations, is rapidly changing under pressure: close to the SDW-SC juncture, $\Theta$ is vanishingly small, indicative of its critical suppression as $P \approx P_{c}$. From $P_{c}$ upward, $\Theta$ raises rapidly and reaches large values at high pressure (Fig. 1. - a).

The anomaly in the temperature dependence of the nuclear relaxation rate, along with its modification under pressure hints at a direct participation of spin fluctuations in linear- $T$ resistivity above $T_{c}$. Low-energy SDW fluctuations evidenced by the low-frequency NMR probe can act as an important source of scattering for electrons and can then influence the resistivity in a significant way. At the same time, the same SDW fluctuations have the ability to promote unconventional d-wave Cooper pairing. This may explain the amazing correlation between the non Fermi liquid features of the normal state and superconductivity whose importance is best meant by the size of $T_{c}$.

\section{Theory of the quasi-one-dimensional electron gas model}

\subsection{Model}

Despite the apparent molecular complexity of (TMTSF) $)_{2} X$ solids, their low energy electronic structure can be approached in a relatively simple way with the aid of molecular orbitals. Dominant van der Waals bonding yields a strongly anisotropic overlap of the highest occupied $\pi$ molecular orbitals, the so-called HOMO's of the TMTSF molecule. The HOMO is partly filled owing to the charge transfer of one electron per two molecules towards the radical $X$, as indicated by the 2:1 stoichiometry of the compound. An extended-Hückel analysis of the electronic structure reveals the presence of a single band crossing the Fermi level [36, 24]. A simple one-component quasi-1D open Fermi surface follows, which consists of two warped planes centered around the one-dimensional Fermi wave vector $\pm k_{F}$ of isolated chains. The TMTSF stacks, however, are not uniform and display a slight dimerisation which 

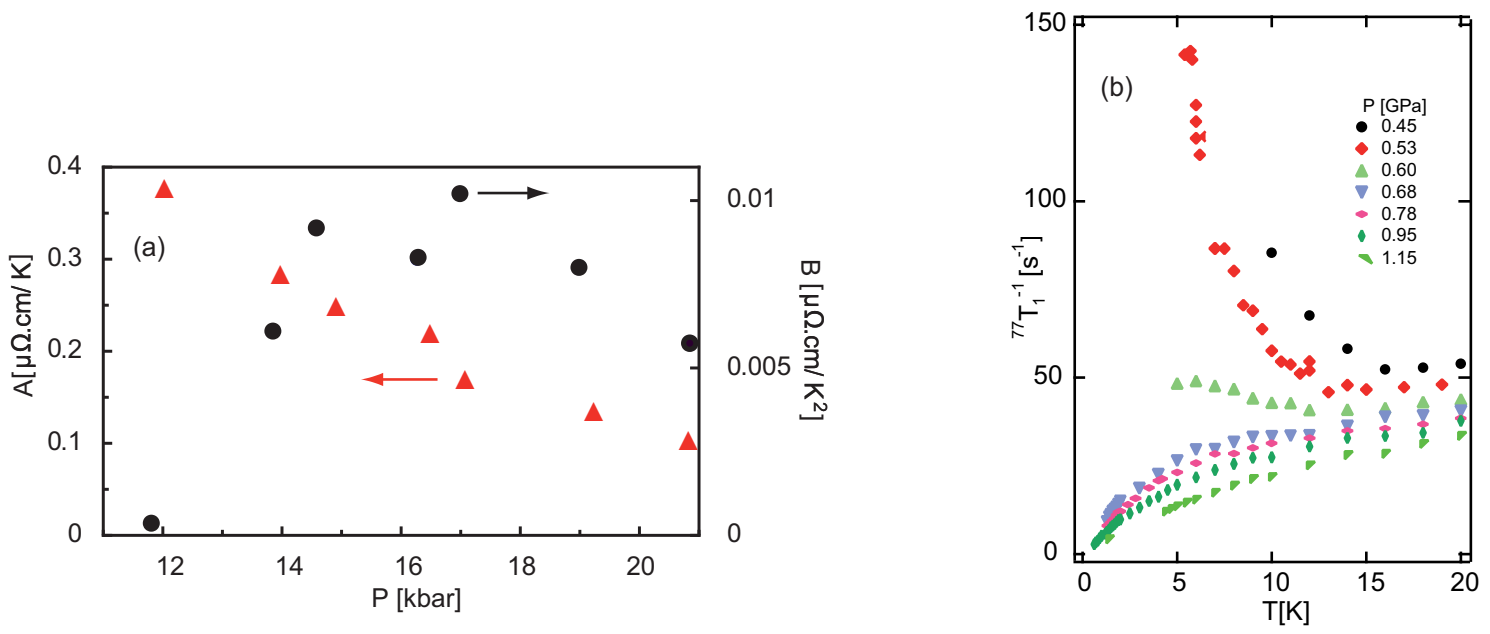

Figure 2: (a) Pressure dependence of the resistivity coefficients $A$ and $B$ of the polynomial fit $\rho_{a}(T)=\rho_{0}+A T+B T^{2}$ of the data of Figure 1 b; after Ref. [18]. (b) Temperature dependence of spin-lattice nuclear relaxation rate $T_{1}^{-1}$ in (TMTSF) ${ }_{2} \mathrm{PF}_{6}$ at different pressures; after ref. [20].

doubles the longitudinal lattice periodicity and then opens a small gap, $\Delta_{D}$, at $\pm 2 k_{F}$, namely in the middle of the 3/4filled band of the uniform limit. There is one hole per dimer in average and the band can be considered as effectively half-filled at low energy or temperature compared to $\Delta_{D}$ [37]. An important outcome of the half-filled character of the band, though weak, is the possibility of electron-electron Umklapp scattering along the stacks. This reinforces the impact of nesting, which is at the core of instabilities of the electron system toward density-wave formation.

A suitable tight-binding quasi-1D model of the energy spectrum is given by

$$
E_{p}(\mathbf{k})=v_{F}\left(p k-k_{F}\right)-2 t_{\perp b} \cos k_{b}-2 t_{\perp b}^{\prime} \cos 2 k_{b}-2 t_{\perp c} \cos k_{c},
$$

for which the longitudinal tight-binding part $-2 t_{a} \cos k$ has been linearized around the 1D Fermi points $\pm k_{F}( \pm \pi / 2)$; here $v_{F}=2 t_{a}\left(E_{F}=v_{F} k_{F}\right)$ corresponds to the longitudinal Fermi velocity (energy) and $p$ refers to right $(p=+)$ and left ( $p=-$ ) moving carriers. The anisotropy in the band structure is about $E_{F} \sim 15 t_{\perp b} \sim 450 t_{\perp c}$, along the $a, b$ and $c$ directions, which are taken orthogonal for simplicity [36, 24]. A next-to-nearest neighbor hopping term of amplitude $t_{\perp b}^{\prime} \ll t_{\perp b}$ along the transverse $b$ direction enters in the definition of $E_{p}(\mathbf{k})$. This term violates the electron-hole symmetry of the spectrum (1), i.e., $E_{p}\left(\mathbf{k}+\mathbf{q}_{0}\right) \neq-E_{-p}(\mathbf{k})$ at the nesting wave vector $\mathbf{q}_{0}=\left(2 k_{F}, \pi, \pi\right)$. Deviations are introduced by a finite $t_{\perp b}^{\prime}$, which simulates the main influence of pressure on nesting.

The partition function of the quasi-1D electron gas model, $Z=\iint \mathfrak{D} \psi^{*} \mathfrak{D} \psi e^{S_{0}+S_{I}}$, when expressed as a functional integral over fermion fields, is parametrized by an action $S$ whose quadratic part, $S_{0}$, for free electrons is given by

$$
S_{0}\left[\psi^{*}, \psi\right]=\sum_{p, \sigma, \bar{k}}\left[G_{p}^{0}(\bar{k})\right]^{-1} \psi_{p, \sigma}^{*}(\bar{k}) \psi_{p, \sigma}(\bar{k}),
$$

where

$$
G_{p}^{0}(\bar{k})=\left[i \omega_{n}-E_{p}(\mathbf{k})\right]^{-1},
$$

is the bare fermion propagator and $\bar{k}=\left(\mathbf{k}, \omega_{n}= \pm \pi T, \pm 3 \pi T, \ldots\right)$. The interacting part $S_{I}$ takes the form

$$
\begin{gathered}
S_{I}=-\frac{1}{T L N_{\perp}} \sum_{\{\bar{k}, \sigma\}}\left[g_{\{\sigma\}}\left(k_{b 1}^{\prime}, k_{b 1}, k_{b 2}\right) \psi_{+, \sigma_{1}^{\prime}}^{*}\left(\bar{k}_{1}^{\prime}\right) \psi_{-, \sigma_{2}^{\prime}}^{*}\left(\bar{k}_{2}^{\prime}\right) \psi_{-, \sigma_{2}}\left(\bar{k}_{2}\right) \psi_{+, \sigma_{1}}\left(\bar{k}_{1}\right)\right. \\
\left.+\frac{1}{2} g_{3}\left(k_{b 1}^{\prime}, k_{b 1}, k_{b 2}\right)\left(\psi_{+, \sigma_{1}}^{*}\left(\bar{k}_{1}^{\prime}\right) \psi_{+, \sigma_{2}}^{*}\left(\bar{k}_{2}^{\prime}\right) \psi_{-, \sigma_{2}}\left(\bar{k}_{2}\right) \psi_{-, \sigma_{1}}\left(\bar{k}_{1}\right)+\text { c.c. }\right)\right] \delta_{\bar{k}_{1}+\bar{k}_{2}=\bar{k}_{1}^{\prime}+\bar{k}_{2}^{\prime}( \pm \bar{G})} .
\end{gathered}
$$

In the g-ology prescription, the electron-electron interaction separates into normal and Umklapp processes. The former part,

$$
g_{\{\sigma\}}\left(k_{b 1}^{\prime}, k_{b 1}, k_{b 2}\right)=g_{2} \delta_{\sigma_{2} \sigma_{2}^{\prime}} \delta_{\sigma_{1} \sigma_{1}^{\prime}}-g_{1} \delta_{\sigma_{1}^{\prime} \sigma_{2}} \delta_{\sigma_{2}^{\prime} \sigma_{1}}
$$




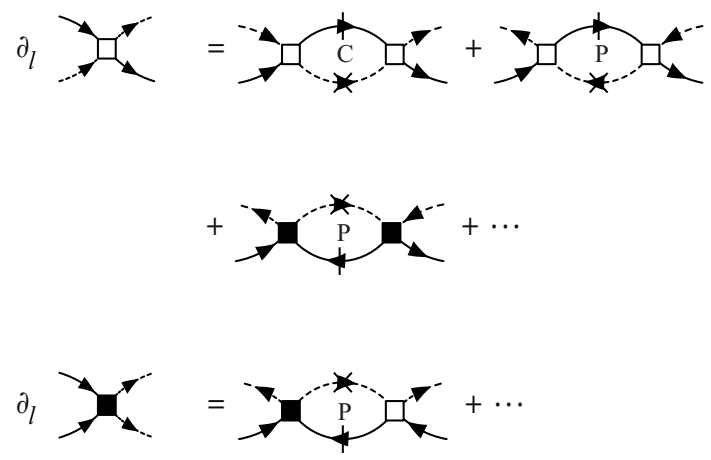

Figure 3: One loop RG flow equations for the normal $g_{1,2}$, (open square) and Umklapp $g_{3}$ (full square) scattering amplitudes. Here $C$ and $P$ stand for the Cooper and Peierls loops. The full (dashed) lines refer to right (left) moving electrons.

retains the backward $\left(g_{1}\right)$ and forward $\left(g_{2}\right)$ bare scattering amplitudes between right and left moving carriers. As for Umklapp scattering, for which $\bar{G}=\left(4 k_{F}, \mathbf{0}\right)$, it is defined by the bare amplitude $g_{3}\left(k_{b 1}^{\prime}, k_{b 1}, k_{b 2}\right)=g_{1} \Delta_{D} / E_{F}$, which is small for weakly dimerized chains. This is the situation that prevails in the Bechgaard salts where one has typically $\Delta_{D} / E_{F} \lesssim 0.1$ [37]. All the above scattering amplitudes are independent of the momentum at the bare level. They are commonly expressed in terms of the one-site and nearest-neighbor site couplings parameters $U$ and $V$ of the extended Hubbard model in the continuum (electron gas) limit, namely $g_{1}=U-2 V, g_{2}=U+2 V$, and $g_{3}=g_{1} \Delta_{D} / E_{F}$.

The range of various band and coupling parameters of the above quasi-1D electron gas model can be fixed from various sources. Band calculations, as well experiments are compatible with the following typical set of values for the kinetic part of the Hamiltonian, $E_{F} \simeq 3000 \mathrm{~K}, t_{\perp b} \simeq 200 \mathrm{~K}$ and $t_{\perp c} \lesssim 10 \mathrm{~K}$. As for the bare amplitude of the $g_{i}{ }^{\prime} s$, the observed enhancement of uniform magnetic susceptibility can be called for to fix the range of the backscattering amplitude $g_{1}$ [34]. The analysis of the temperature dependence of susceptibility is consonant with a value revolving around $\tilde{g}_{1} \equiv g_{1} / \pi v_{F} \sim 0.3$ (henceforth normalized by the longitudinal bandwidth). Given the size of the dimerization gap $\Delta_{D}$, this fixes the amplitude of Umklapp scattering at a small, but finite value, $\tilde{g}_{3} \simeq 0.02$. Finally, the bare $\tilde{g}_{2}$ can be estimated by the value needed to match the optimal $T_{\mathrm{SDW}}$ observed to the calculated scale, as obtained from RG with the above set of figures in the limit of small $t_{\perp b}^{\prime}$. Thus the maximum $T_{\text {SDW }}^{0}(\sim 25 \mathrm{~K})$ found on experimental grounds in the cousin compounds (TMTTF) $)_{2} X$ [38], yields $\tilde{g}_{2} \sim 0.6$.

\subsection{One-loop renormalisation group results}

Long-distance correlations and the propensity for ordering in the most conducting $-a b$ - plane can be studied by the RG method [39, 40, 16]. In this approach, one proceeds in the partition function $Z$ to the successive integration of electronic degrees of freedom $\psi^{(*)}$, from the high energy cutoff $E_{F}$ down to the energy distance $E_{F} e^{-\ell}$ above and below each Fermi sheet at step $\ell \geq 0$. Constant energy surfaces at $\ell$ are divided into a number patches [41, 40], each being indexed by a particular $k_{b}$ value for the momentum along $b$.

At the one-loop level, the partial integration leads to successive corrections to the scattering amplitudes $\tilde{g}_{i}$ as function of $\ell$ and for a given temperature $T$. These come from the logarithmically singular loops of particle-particle (Cooper) and particle-hole (Peierls) scattering channels. Both contributions generate momentum dependence for the couplings, which is retained for the $b$ direction only. This leads to the flow equations of Fig. 3 [40, 39], which are written in the schematic form

$$
\begin{aligned}
\partial_{\ell} \tilde{g}_{i=1,2}\left(k_{b 1}^{\prime}, k_{b 2}, k_{b 1}\right) & =\sum_{n, n^{\prime}=1}^{3}\left\{\epsilon_{C, i}^{n, n^{\prime}}\left\langle\tilde{g}_{n} \cdot \tilde{g}_{n^{\prime}} \cdot \partial_{\ell} \mathcal{L}_{C}\right\rangle_{k_{b}}+\epsilon_{P, i}^{n, n^{\prime}}\left\langle\tilde{g}_{n} \cdot \tilde{g}_{n^{\prime}} \cdot \partial_{\ell} \mathcal{L}_{P}^{\prime}\right\rangle_{k_{b}}\right\}, \\
\partial_{\ell} \tilde{g}_{3}\left(k_{b 1}^{\prime}, k_{b 2}, k_{b 1}\right) & =\sum_{n=1}^{2} \epsilon_{P, 3}^{3, n}\left\langle\tilde{g}_{3} \cdot \tilde{g}_{n} \cdot \partial_{\ell} \mathcal{L}_{P}\right\rangle_{k_{b}},
\end{aligned}
$$

where the momentum dependence of various products has been masked for simplicity. Here the Cooper and Peierls loops $\mathcal{L}_{P}\left(k_{b}, q_{P}, t_{b}^{\prime}\right)=T \sum_{\omega_{n}} \int d E_{P}\left[G_{p}^{0}(\bar{k})\right]_{\ell}\left[G_{-p}^{0}\left(\bar{k}-\bar{q}_{P}\right)\right]_{\times}$and $\mathcal{L}_{C}\left(k_{b}, q_{C}\right)=T \sum_{\omega_{n}} \int d E_{P}\left[G_{p}^{0}(\bar{k})\right]_{\ell}\left[G_{-p}^{0}\left(-\bar{k}+\bar{q}_{C}\right)\right]_{\times}$ 
are evaluated at $q_{P}=k_{b 1}-k_{b 1}^{\prime}$ and $q_{C}=k_{b 1}+k_{b 2}$, respectively. These are expressed as integrals over products of propagators on $\left(\left[G_{-p}^{0}\right]_{\ell}\right)$ and above $\left(\left[G_{-p}^{0}\right]_{\times}\right)$the energy shell at $E_{F} e^{-\ell}$. Here $\langle\ldots\rangle_{k_{b}}$ stands for an average over $k_{b}$ and $\epsilon_{C, P, i}^{n n^{\prime}}$ are the coefficients that fix the sign of closed loops $\left(\epsilon_{P, i}^{n n^{\prime}}=-2\right)$, vertex corrections $\left(\epsilon_{P, i}^{n n^{\prime}}=1\right)$ and ladder graphs $\left(\epsilon_{C, i}^{n n^{\prime}}=-\epsilon_{P, i}^{n n^{\prime}}=-1\right)$. These different contributions will then interfere with one another as a function of $\ell$ or the energy distance $E_{F} e^{-\ell}$ from the Fermi surface. For small $\Delta_{D}$ and then weak initial Umklapp term, interference for high energy - 1D - degrees of freedom where $E_{F} e^{-\ell}>t_{b}$, give rise to coupling constant flows differing very little from those obtained in the 1D (Luttinger liquid) limit. As $E_{F} e^{-\ell}<t_{b}$, however, sensitivity to the wrapping of the Fermi surface develops and interference modifies accordingly, becoming non uniform along the Fermi surface. The $g^{\prime} s$ then acquire a $k_{b}$ dependence and their flows can be governed by a strong coupling fixed point where the $g^{\prime} s$ become singular, signaling an instability of the metallic state against ordering.

For the above model of a quasi-1D metal with repulsive intrachain interactions and weak dimerization, long-range order can only occur in the SDW and d-wave SC channels [40, 39], as shown by a singularity in the corresponding static response functions denoted by $\chi_{\mathrm{SDw}}\left(\mathbf{q}_{0}\right)$ and $\chi_{\mathrm{SCd}}(0)$, respectively. In the RG framework, we have

$$
\chi_{\mu}\left(\mathbf{q}_{\mu, 0}\right)=\left(\pi v_{F}\right)^{-1} \int_{\ell}\left\langle f_{\mu}\left(k_{b}\right) z_{\mu}^{2}\left(k_{b}\right)\right\rangle_{k_{b}} d \ell
$$

whose $k_{b}$ average expression depends on the vertex renormalisation factors $z_{\mu}\left(k_{b}\right)$ and the form factors $f_{\mathrm{SDW}}=1$ and $f_{\mathrm{SCd}}=2 \cos ^{2} k_{b}$ for the SDW and SCd channels, respectively [40, 39]. The $z_{\mu}^{\prime}$ s obey the flow equation

$$
\partial_{\ell} z_{\mu}\left(k_{b}\right)=\left\langle\left(\partial_{\ell} \mathcal{L}_{\mu}\right) \tilde{g}_{\mu} z_{\mu}\left(k_{b}^{\prime}\right)\right\rangle_{k_{b}^{\prime}}
$$

which is governed by the combinations of momentum dependent couplings $\tilde{g}_{\mathrm{SDW}}=\tilde{g}_{2}\left(k_{b}, k_{b}^{\prime}, k_{b}+\pi\right)+\tilde{g}_{3}\left(k_{b}, k_{b}^{\prime}+\right.$ $\left.\pi, k_{b}+\pi\right)$ and $\tilde{g}_{\mathrm{SCd}}=-\tilde{g}_{1}\left(-k_{b}^{\prime}, k_{b},-k_{b}\right)-\tilde{g}_{2}\left(-k_{b}^{\prime},-k_{b}, k_{b}\right)$. A singularity in $\tilde{g}_{\mathrm{SDW}}$ (resp., $\left.\tilde{g}_{\mathrm{SCd}}\right)$ is synonymous with a singularity in $\chi_{\mathrm{SDW}}$ (resp., $\left.\chi_{\mathrm{SCd}}\right)$ at the ordering temperature $T_{\mathrm{SDW}}\left(\right.$ resp., $\left.T_{\mathrm{SCd}}\right)$.

\subsection{Phase diagram, pairing and spin correlations}

From the solution of Eqs. (6) and (8) and the initial conditions of the model, one can follow the evolution of the ordering scale $T_{\mu}$ as a function of the antinesting parameter $t_{\perp b}^{\prime}$ simulating the influence of pressure. In the calculated phase diagram of Fig. 4 $\mathrm{a}$, we observe that at small $t_{\perp b}^{\prime}$, nesting is weakly affected and an instability toward the formation of a SDW state follows at the wave vector $\mathbf{q}_{0}=\left(2 k_{F}, \pi\right)$ and temperature $T_{\mathrm{SDW}} \sim 20 \mathrm{~K}$, which is of the order found in systems like (TMTSF) ${ }_{2} X$ in normal pressure conditions [2, 3, 38]. It should be stressed, here, that the above one-loop calculation yields a $T_{\mathrm{SDW}}$ that is strongly reduced compared to the mean-field limit for which only the singularity of the Peierls scattering channel is retained. This reduction is caused by the influence of the Cooper channel in (6), whose primary interfering effect is to prevent degrees of freedom in the 1D energy range $\left(E_{F} e^{-\ell}>t_{\perp b}\right)$ to produce any instability of the electron gas, in line with the absence of long-range order in one dimension.

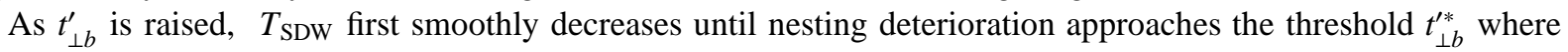
$T_{\text {SDW }}$ falls sharply (Fig. 4 - a). However, the $T_{\text {SDW }}$ instability line, instead of dropping toward zero - in which case it would lead to a quantum critical point - terminates at the beginning of a different transition line, $T_{c}$, marked by a singularity in the SCd response $\chi_{\mathrm{SCd}}$ of the superconducting channel. The quantum critical point is therefore avoided by the occurrence of superconductivity. As for the amplitude of $T_{c}$, it is maximum at the juncture with the SDW phase, giving a ratio $T_{\mathrm{SDW}}^{0} / T_{c}^{0} \sim 20$ between the optimal $T_{\mathrm{SDW}}^{0}$ at zero $t_{\perp b}^{\prime}$ and the maximum $T_{c}$ at $t_{\perp b}^{\prime *}$. Combining these results with those obtained beyond $t_{\perp b}^{\prime *}$ where $T_{c}$ is steadily decreasing, yields the phase diagram of Figure 4-a. Singular attractive Cooper pairing is here fed by strong SDW correlations through the interference present in Eqs. 6. The d-wave character of the superconducting transition marks the onset of a SCd order parameter $\Delta\left(k_{\perp b}\right)=\Delta \cos k_{\perp b}+O\left(\cos 2 k_{\perp b}\right)$, which indicates that singlet Cooper pairs are essentially formed by electrons of neighboring chains [42, 43]. Identifying in first approximation the antinesting parameter $t_{\perp b}^{\prime}$ as being proportional to real pressure, the essential features of the calculated RG phase diagram of Fig. 4 4 a compare favorably with the sequence of transitions found on experimental grounds (Fig. 11-a) [2, 17].

The impact of interfering SDW-SCd correlations is not limited to the sequence of instabilities lines, but its influence extends also in the normal phase. This is particularly noticeable when one considers the temperature dependence of SDW susceptibility in the superconducting sector of the phase diagram [16]. Insert of Fig. 44-b shows for example 

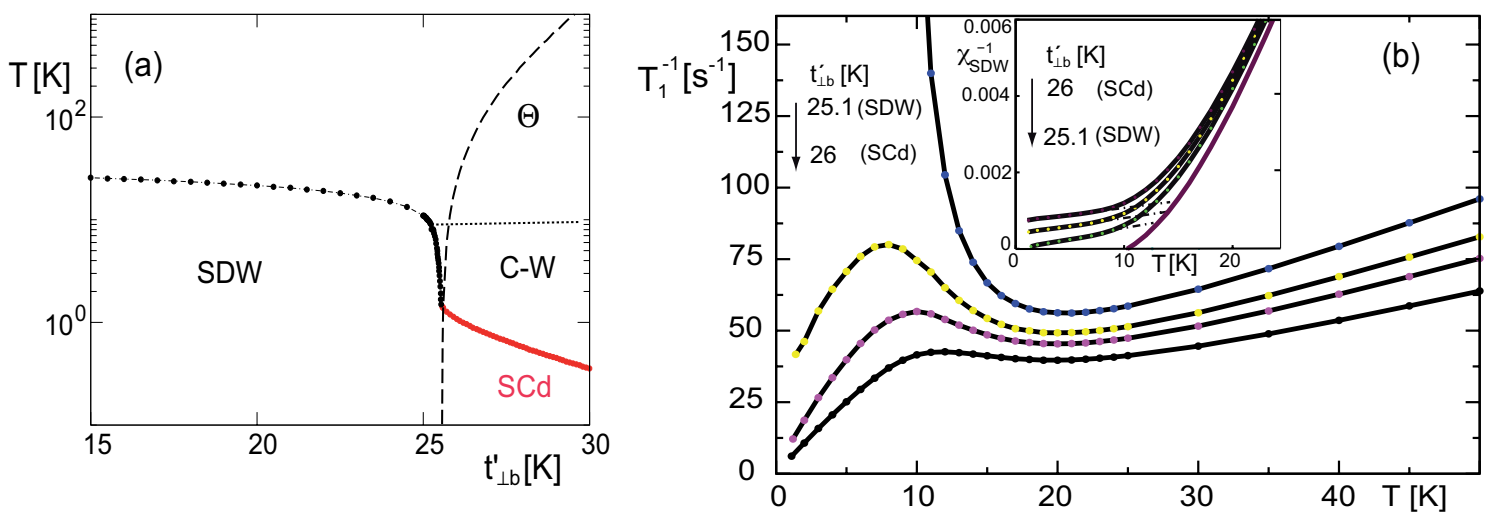

Figure 4: (a) One loop RG phase diagram of the quasi-one-dimensional electron gas model versus the 'pressure' antinesting parameter $t_{\perp b}^{\prime}$; also shown the variation of the Curie-Weiss $(\mathrm{C}-\mathrm{W})$ temperature scale $\Theta$ and the $\mathrm{C}-\mathrm{W}$ emperature domain. (b) Calculated nuclear spin-lattice relaxation rate $T_{1}^{-1}$ versus temperature for different $t_{\perp b}^{\prime}$. Inset: temperature dependence of the inverse SDW susceptibility $\left(\pi v_{F} \chi_{\mathrm{SDW}}\right)^{-1}$ versus temperature showing the C-W behavior at low temperature (dashed lines). After Ref. [16].

that $\chi_{\mathrm{SDW}}\left(\mathbf{q}_{0}\right)$ still increases at the approach of $T_{c}$, showing the Curie-Weiss enhancement $\chi_{\mathrm{SDW}}\left(\mathbf{q}_{0}\right) \propto(T+\Theta)^{-1}$ below $10 \mathrm{~K}$ or so. The origin of the enhancement comes from the positive feedback of SCd Cooper pairing on SDW correlations, which reinforces staggered spin fluctuations over a Curie-Weiss temperature interval of about ten times $T_{c}$. The Curie-Weiss scale $\Theta$ for SDW fluctuations displays a particular variation with 'pressure' $t_{\perp b}^{\prime}$ : it starts from zero at $t_{\perp b}^{* *}$ and then raises rapidly as $t_{\perp b}^{\prime}>t_{\perp b}^{\prime *}$, showing that SDW fluctuations decreases in amplitude, which in turn correlated with the decline of $T_{c}$. Considering the point $t_{\perp b}^{\prime *}$ at zero temperature as quantum critical in the absence of superconductivity, $\Theta$ can be seen as a characteristic energy scale for SDW fluctuations that increases to some power of the tuning parameter $t_{\perp b}^{\prime}$. In the usual terminology of quantum phase transitions, one can write $\Theta \sim \xi^{-z}$, where $\xi \sim\left(t_{\perp b}^{\prime}-t_{\perp b}^{\prime *}\right)^{-v}$ is the zero temperature SDW correlation length close to $t_{\perp b}^{\prime *}$. In the one-loop approximation, the exponents are $v=1 / 2$ for the correlation length and $z=2$ for the dynamics [16], which leads to a linear variation of $\Theta$ with $t_{\perp b}^{\prime}$ in the vicinity of $t_{\perp b}^{*}$.

\subsection{Nuclear relaxation rate}

The Curie Weiss behavior of $\chi_{\text {SDW }}$ has also an effect on the temperature dependence of other quantities sensitive to staggered spin fluctuations. This is the case of the NMR spin-lattice relaxation rate $T_{1}^{-1}$, whose expression can be written as an integral of the imaginary part of the spin response over all wave vectors $\mathbf{q}$,

$$
T_{1}^{-1}=T \int\left|A_{\mathbf{q}}\right|^{2} \frac{\chi^{\prime \prime}(\mathbf{q}, \omega)}{\omega} d^{d} q .
$$

The $T_{1}^{-1}$ calculation using the above one-loop RG results [16], leads to

$$
T_{1}^{-1} \approx c_{0} T+c_{1} T \frac{\chi \mathrm{SDW}\left(\mathbf{q}_{0}\right)}{\sqrt{1+\xi_{c}^{2}}}
$$

where $\xi_{c}(\propto \sqrt{\chi \mathrm{SDW}})$ is the SDW correlation length along the third direction; $c_{0}$ and $c_{1}$ are positive constants. The expression for $T_{1}^{-1}$ then superimposes a linear- $T$ Korringa like contribution coming from uniform $(\mathbf{q} \sim 0)$ spin fluctuations, which dominates at large temperature, and an anomalous one coming from SDW $\left(\mathbf{q} \sim \mathbf{q}_{0}\right)$ fluctuations, which eventually takes over at lower temperature. In the SDW sector of the phase diagram where both $\chi_{\text {SDW }}$ and $\xi_{c}$ become singular at $T_{\mathrm{SDW}}, T_{1}^{-1} \propto\left(T-T_{\mathrm{SDW}}\right)^{-\frac{1}{2}}$ develops a square root singularity (Fig. 4 $\mathrm{b}$ ), a characteristic of a 3D critical behavior known to be consonant with the experimental situation of Fig. 22 [20, 34]. As one reaches the superconducting region, from $t_{\perp b}^{\prime *}$ upwards in the phase diagram, $T_{1}^{-1}$, albeit non longer singular, is strongly enhanced signaling marked deviations from the linear- $T$ Korringa behavior predicted for a Fermi liquid. The anomaly extends deeply in the 2D metallic phase $\left(\xi_{c} \ll 1\right)$, up to at least $20 T_{c}$. According to $[10)$, the amplitude of the enhancement is correlated 


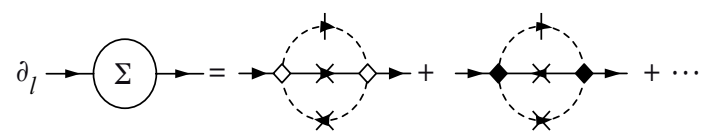

Figure 5: Two-loop RG flow equation for the one-particle self-energy $\Sigma_{+}$.

to the one of SDW fluctuations and in turn to the size of $T_{c}$ as a function of $t_{\perp b}^{\prime}$. The Curie-Weiss behavior of $\chi_{\mathrm{SDW}}$ is found for $\left(T_{1} T\right)^{-1} \sim(T+\Theta)^{-1}$ as well, so that the scale $\Theta$ extracted from the $T_{1}^{-1}$ data (Fig. 2. b) [20, 19, 22] coincides with the one governing the behavior of $\chi_{\mathrm{SDW}}$ in the present theory. The interference induced reinforcement of SDW correlations by SCd Cooper pairing thus appears as the mechanism responsible for the Curie-Weiss behavior observed in $T_{1}^{-1}$ [16].

\subsection{Quasi-particle scattering rate and resistivity}

Carrying out the RG transformation up to the two-loop level introduces a renormalization of the inverse oneparticle propagator $\left[G_{p}^{0}\right]^{-1} \rightarrow\left[G_{p}^{0}\right]^{-1}-\Sigma_{p}$, in the form of Matsubara self-energy corrections $\Sigma_{p}$ (Fig. 5). On the Fermi surface $\mathbf{k}_{F}\left(k_{b}\right)$, the self-energy takes the form

$$
\Sigma_{p}\left(\bar{k}_{b}\right)=i \omega_{n}\left[1-z\left(\bar{k}_{b}\right)\right]-z_{\perp}\left(\bar{k}_{b}\right)
$$

where $z$ and $z_{\perp}$ are renormalization factors evaluated at $\bar{k}_{b}=\left(\mathbf{k}_{F}\left(k_{b}\right), \omega_{n}\right)$. The corresponding flow equations obtained in Ref. [44], can be written schematically as

$$
\begin{aligned}
\partial_{\ell} \ln z\left(\bar{k}_{b}\right) & =\sum_{i=1}^{3} \sum_{n, n^{\prime}}\left\langle\tilde{g}_{n} \cdot \tilde{g}_{n^{\prime}} \cdot \partial_{\ell} \mathcal{T}_{i}\right\rangle_{k_{b}^{\prime}, q_{b}^{\prime}}, \\
\partial_{\ell} z_{\perp}\left(\bar{k}_{b}\right) & =z^{-1}\left(\bar{k}_{b}\right) \sum_{i=1}^{3} \sum_{n, n^{\prime}}\left\langle\tilde{g}_{n} \cdot \tilde{g}_{n^{\prime}} \cdot \partial_{\ell} \mathcal{T}_{\perp, i}\right\rangle_{k_{b}^{\prime}, q_{b}^{\prime}},
\end{aligned}
$$

where $\mathcal{T}_{i,(\perp)}= \pm T^{2} \sum_{\{\omega\}} \int_{\mathcal{D}_{i, \perp)}} d E_{p}\left[G_{p}^{0}\right]_{\ell}\left[G_{-p}^{0}\right]_{\times}\left[G_{-p}^{0}\right]_{\times}$corresponds to the expressions of the two-loop diagrams of Figure 5] whose sign is fixed by their topology. Solving the equations for $z$ and $z_{\perp}$, consistently with those at the one-loop level for the $\tilde{g}_{n}$, yields $\Sigma_{p}\left(\bar{k}_{b}\right)$ on the Fermi surface for all $\omega_{n}$. Using a Padé procedure, the Matsubara self-energy can be analytically continued to the retarded form of self-energy, which consists of a real $\left[\Sigma_{p}^{\prime}\left(\mathbf{k}_{F}\left(k_{b}\right), \omega\right)\right]$ and imaginary $\left[\Sigma_{p}^{\prime \prime}\left(\mathbf{k}_{F}\left(k_{b}\right), \omega\right)\right]$ parts at real frequency $\omega$. These two ingredients enter in the determination of spectral properties of the quasi-1D electron gas model [44].

Among quantities of interest that can be extracted from the imaginary part is the electron-electron scattering rate on the Fermi surface

$$
\tau^{-1}\left(\mathbf{k}_{F}\left(k_{b}\right)\right)=-2 \Sigma^{\prime \prime}\left(\mathbf{k}_{F}\left(k_{b}\right), \omega \rightarrow 0\right)
$$

In the relaxation time approximation for conductivity, the average scattering rate $\langle\tau\rangle_{k_{b}}^{-1}$ for an open, strongly anisotropic, Fermi surface will give the contribution of the singular scattering channels to the temperature dependence of electrical resistivity. By excluding the impurity contribution, the latter reads

$$
\Delta \rho=\frac{4 \pi}{\omega_{p}^{2}}\langle\tau\rangle_{k_{b}}^{-1},
$$

where $\omega_{p}$ is the plasma frequency, e.g., along the chains. The calculated low temperature dependence of $\langle\tau\rangle_{k_{b}}^{-1}$ down to $T_{c}$ is shown in Fig. 6-a, as a function of $T_{c}\left(t_{\perp b}^{\prime}\right)$ in the superconducting region of the phase diagram of Fig. 4 4 - a. Close to the critical point $t_{\perp b}^{*}$, where $T_{c}$ approaches its maximum, the behavior of $\langle\tau\rangle_{k_{b}}^{-1}$, though metallic, does not follow the $T^{2}$ law expected for a Fermi liquid above $T_{c}$. A strict linear behavior, $\langle\tau\rangle_{k_{b}}^{-1}=a T$, is instead seen up to four times $T_{c}$ or so, namely within the temperature range where the Curie-Weiss behavior takes place for $\chi_{\text {SDW }}$ and $T_{1}^{-1}$. We have seen that by moving toward $t_{\perp b}^{\prime *}$ from above, SDW correlations, stimulated by Cooper pairing, grow in amplitude ; the characteristic energy scale of spin fluctuations then decreases to ultimately attain thermal energy. In two dimensional 

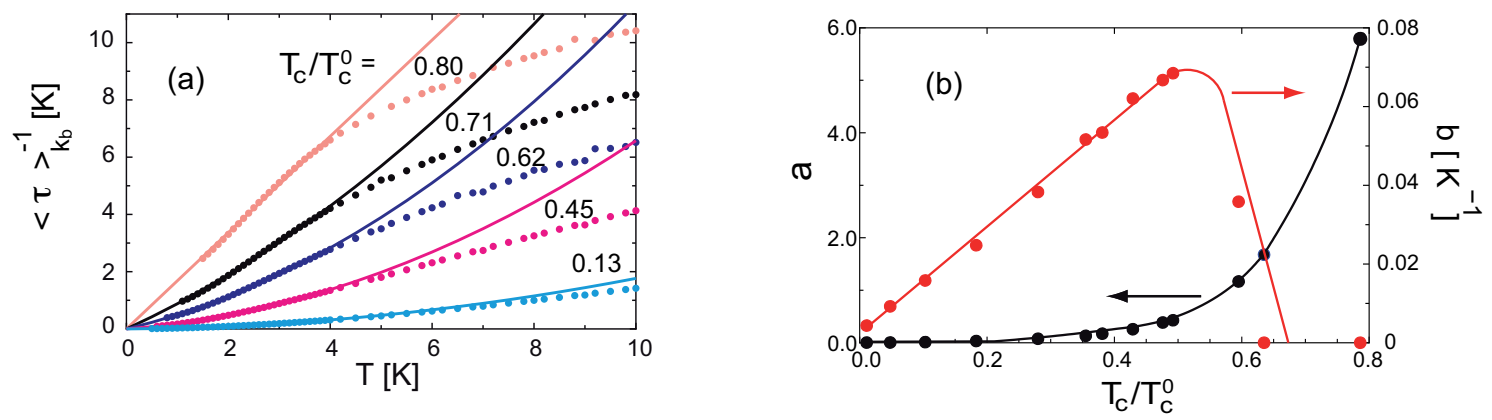

Figure 6: (a) Averaged electron-electron scattering rate versus temperature calculated for different superconducting $T_{c}$ of the RG phase diagram (Fig. 3. a). The continuous lines correspond to the polynomial fit $\langle\tau\rangle_{k_{b}}^{-1}=a T+b T^{2}$ below $4 \mathrm{~K}$. (b) Variation of the coefficients for a linear-T and Fermi liquid terms of the polynomial fit versus $T_{c}$.

systems, these conditions are particularly favorable for the emergence of a linear $T$-behavior in the scattering rate [45], which the above two-loop RG calculations confirm. By treating density-wave and Cooper pairings on the same footing, the RG also provides an explanation of the origin of the linear $T$-resistivity term seen in compounds like the Bechgaard salts (Fig.1 1.b), and shows how interfering orders connect the amplitude $a$ to $t_{\perp b}^{\prime}$ and in turn to the size of $T_{c}$.

Moving away from the SDW-SCd juncture along the $t_{\perp b}^{\prime}$ axis, the $\langle\tau\rangle_{k_{b}}^{-1}$ temperature dependence starts to display some upward curvature [Fig. 6]. It turns out that within a temperature interval of at least four times $T_{c}$, the overall temperature dependence is adequately fitted by the polynomial expression

$$
\langle\tau\rangle_{k_{b}}^{-1} \approx a T+b T^{2},
$$

which can be interpreted as the superimposition of two scattering channels for electrons under 'pressure'. The onset of a Fermi liquid component in the scattering rate results from the continuing stiffening of spin fluctuations, whose spectral weight moves to higher frequencies, as shown for example by the evolution of $\Theta$ in nuclear relaxation rate under 'pressure' (Fig.4-b). The scattering of electrons on higher energetic modes compared to temperature favors the onset of a $T^{2}$ component in $\langle\tau\rangle_{k_{b}}^{-1}[45,46]$. In Fig. 66-b, the variation of the linear and quadratic coefficients $a$ and $b$ is shown as a function of the ratio $T_{c} / T_{c}^{0}$ for Cooper pairing tied by interference to the strength of SDW correlations. When $T_{c} / T_{c}^{0} \lesssim 1$, namely for $t_{\perp b}^{\prime} \gtrsim t_{\perp b}^{\prime *}, b$ is vanishingly small and the behavior of the scattering rate is essentially linear in temperature. However, as $T_{c}$ decreases, $a$ falls approximately as $a \sim T_{c}^{2}$, while $b$ initiates a steep growth reaching a maximum at intermediate $T_{c} / T_{c}^{0}$ to finally decrease substantially in the limit of small $T_{c}$. Such a behavior for $b$ vs $T_{c}$ results from the balance between the rapid increase of the characteristic energy scale of SDW fluctuations (compared to temperature) on the one hand, and the decrease in the amplitude of the same fluctuations on the other [Fig.4]. The former is responsible for the presence of a Fermi liquid component, whereas the latter governs the decline of both the Fermi liquid and linear contributions over the entire range of $t_{\perp b}^{\prime}$ where $T_{c}$ takes appreciable values. The fact that Cooper pairing reinforces SDW correlations by interference, makes existence of a linear component not limited to the very close proximity of $t_{\perp b}^{\prime *}$, as it would be expected for a quantum critical point, but is stretched out over the whole interval where $T_{c}$ is discernible.

The features depicted by $a$ and $b$ in Fig. 6 6 b find a favorable echo in electrical resistivity of (TMTSF) ${ }_{2} \mathrm{PF}_{6}$ under pressure (Figs. 1-b and 2-a). The results also supply a direct connection between scattering and spin fluctuations on one hand, and Cooper pairing on the other. 


\section{Summary and conclusion}

Antiferromagnetism bordering on superconductivity certainly stands out as the most representative feature of the phase diagram of the Bechgaard salts series of organic conductors under pressure. Far from being unrelated the two types of orders prove to be intimately connected, a connection shown to be suitably formulated by the weak coupling renormalization group theory of the repulsive quasi-one-dimensional electron gas model. At the heart of this formulation resides an accurate integration of the two interfering nesting and Cooper pairing mechanisms, which condition the instability of the metallic state towards the onset of long-range order. By tuning the amplitude of nesting alterations, interference controls the sequence of spin-density-wave and d-wave superconducting instabilities, a structure that matches fairly well the one displayed by the phase diagram of the (TMTSF) ${ }_{2} X$. Mixed pairings not only exert influence on instability lines, but also affect short-range spin correlations whose amplitude is strengthened by Cooper pairing in the normal state. Mutual pairing strengthening results in the Curie-Weiss enhancement of staggered spin fluctuations which governs, for instance the temperature dependence of the nuclear spin-lattice relaxation rate above $T_{c}$, in agreement with experimental situation in the Bechgaard salts. The mark left by spin fluctuations can be found in other quantities like the electron-electron scattering rate which is relevant for the temperature dependence of electrical resistivity. It was shown that it is within the Curie-Weiss domain of spin correlations that a linear- $T$ dependence for the scattering rate takes place. Its amplitude correlates with the strength of Cooper pairing or $T_{c}$, in line with the evolution of linear- $T$ resistivity seen under pressure in the Bechgaard salts.

The adherence of the above ideas with experimental facts indicates that the problem raised by magnetism and Cooper pairing in unconventional superconductors like the Bechgaard salts can be approached in a unified fashion by means of a weak coupling scaling theory. From a broader viewpoint, this brings us to ask in conclusion whether the same mechanisms are at play in other series of higher $T_{c}$ unconventional superconductors for which striking similarities with the Bechgaard salts can be found.

The authors acknowledges fruitful and continuous collaboration with P. Auban-Senzier, D. Bergeron, N. DoironLeyraud, D. Jérome, S. René de Cotret and L. Taillefer. C. B. thanks S. E. Brown for many useful discussions on the topics developed in this work. This work has received the financial support from the National Science and Engineering Research Council of Canada (NSERC), Réseau Québcois des Matériaux de Pointe (RQMP) and the Quantum materials program of Canadian Institute of Advanced Research (CIFAR). The authors are also thankful to the Réseau Québécois de Calcul Haute Performance (RQCHP) for supercomputer facilities at the Université de Sherbrooke.

\section{References}

[1] D. Jérome, A. Mazaud, M. Ribault, K. Bechgaard, J. Phys. (Paris) Lett. 41 (1980) L95.

[2] D. Jérome, H. J. Schulz, Adv. Phys. 31 (1982) 299.

[3] C. Bourbonnais, D. Jérome, in: A. Lebed (Ed.), The Physics of Organic Superconductors and Conductors, volume 110, Springer Series in Materials Science, Springer, Heidelberg, 2008, p. 357. ArXiv:cond-mat/0904.0617.

[4] F. Steglich, J. Aarts, W. L. C.D. Bredl, D. Meschede, W. Franz, H. Schäfer, Phys. Rev. Lett. 43 (1979) 1892.

[5] N. D. Mathur, F. M. Grosche, S. R. Julian, I. R. Walker, D. M. Freye, R. K. W. Haselwimmer, G. G. Lonzarich, Nature 394 (1998) 39.

[6] J. Bednorz, K. Muller, Z. Phys. B 64 (1986) 189.

[7] L. Taillefer, Annu. Rev. Condens. Matter Phys. 1 (2010) 51.

[8] N. P. Armitage, P. Fournier, R. L. Greene, Rev. Mod. Phys. 82 (2010) 2421.

[9] V. N. Laukhin, E. E. Kostyuchenko, Y. V. Sushko, I. F. Schegolev, E. B. Yagubskii, JETP Lett. 41 (1985) 81.

[10] H. Urayama, H. Yamochi, G. saito, K. Nozawa, T. Sugano, M. Kinoshita, S. Sato, K. Oshima, A. Kawamoto, J. Tanaka, Chem. Lett. (1988) 55.

[11] K. Kanoda, Physica C 282-287 (1997) 299.

[12] S. Lefebvre, P. Wzietek, S. Brown, C. Bourbonnais, D. Jérome, C. Mzire, M. Fourmigu, P. Batail, Phys. Rev. Lett. 85 (2000) 5420.

[13] B. J. Powell, R. H. McKenzie, J. Phys.: Condens. Matter 18 (2006) R827.

[14] Y. Kamihara, T. Watanabe, M. Hirano, H. Hosono, J. Am. Chem. Soc. 130 (2008) 3296.

[15] L. Fang, H. Luo, P. Cheng, Z. Wang, Y. Jia, G. Mu, B. Shen, I. I. Mazin, L. Shan, C. Ren, H. H. Wen, Phys. Rev. B. 80 (2009) 140508(R).

[16] C. Bourbonnais, A. Sedeki, Phys. Rev. B 80 (2009) 085105.

[17] N. Doiron-Leyraud, P. Auban-Senzier, S. R. de Cotret, C. Bourbonnais, D. Jérome, K. Bechgaard, L. Taillefer, Phys. Rev. B 80 (2009) 214531.

[18] N. Doiron-Leyraud, P. Auban-Senzier, S. R. de Cotret, C. Bourbonnais, A. Sedeki, D. Jérome, K. Bechgaard, L. Taillefer, Eur. Phys. J. B 78 (2010) 23.

[19] W. Wu, P. M. Chaikin, W. Kang, J. Shinagawa, W. Yu, S. E. Brown, Phys. Rev. Lett. 94 (2005) 097004. 
[20] S. E. Brown, P. M. Chaikin, M. J. Naughton, in: A. Lebed (Ed.), The Physics of Organic Superconductors and Conductors, volume 110, Springer Series in Materials Science, Springer, Heidelberg, 2008, p. 49.

[21] K. Bechgaard, C. Jacobsen, K. Mortensen, H. Pedersen, N. Thorup, Solid State Comm. 33 (1980) 1119.

[22] F. Creuzet, C. Bourbonnais, L. G. Caron, D. Jérome, A. Moradpour, Synth. Met. 19 (1987) 277.

[23] T. Takahashi, Y. Maniwa, H. Kawamura, G. Saito, Physica 143B (1986) 417.

[24] L. Ducasse, A. Abderraba, J. Hoarau, M. Pesquer, B. Gallois, J. Gaultier, J. Phys. C 19 (1986) 3805.

[25] T. Vuletic, P. Auban-Senzier, C. Pasquier, S. Tomic, D. Jerome, M. Heritier, K. Bechgaard, Eur. Phys. J. B 25 (2002) 319.

[26] I. J. Lee, P. M. Chaikin, M. J. Naughton, Phys. Rev. Lett. 88 (2002) 207002.

[27] J. Shinagawa, Y. Kurosaki, F. Zhang, C. Parker, S. E. Brown, D. Jérome, K. Bechgaard, J. B. Christensen, Phys. Rev. Lett. 98 (2007) 147002.

[28] N. Joo, P. Auban-Senzier, C. R. Pasquier, D. Jérome, K. Bechgaard, Europhys. Lett. 72 (2005) 645.

[29] 2011. S. Yonezawa et al. to be published.

[30] S. Kasahara, T. Shibauchi, K. Hashimoto, K. Ikada, S. Tonegawa, R. Okasaki, H. Shishido, H. Ikeda, H. Takeya, K. Hirata, T. Terashima, Y. Matsuda, Phys. Rev. B 81 (2010) 18519.

[31] A. P. MacKenzie, S. R. Julian, D. C. Sinclair, C. T. Lin, Phys. Rev. B 53 (1996) 5848.

[32] M. Abdel-Jawad, M. P. Kenett, A. C. L. Balicas, A. P. Mackenzie, R. H. Mackenzie, N. E. Hussey, Nature Phys. 2 (2006) 821.

[33] L. Taillefer, Nature Phys. 8 (2006) 209.

[34] P. Wzietek, F. Creuzet, C. Bourbonnais, D. Jérome, K. Bechgaard, P. Batail, J. Phys. I 3 (1993) 171.

[35] C. Bourbonnais, F. Creuzet, D. Jérome, K. Bechgaard, A. Moradpour, J. Phys. (Paris) Lett. 45 (1984) L755.

[36] P. M. Grant, J. Phys. (Paris) Coll. 44 (1983) 847.

[37] V. J. Emery, R. Bruinsma, S. Barisic, Phys. Rev. Lett. 48 (1982) 1039.

[38] B. J. Klemme, S. Brown, P. Wzietek, P. B. G. Kriza, D. Jérome, J.-M. Fabre, Phys. Rev. Lett. 75 (1995) 2408.

[39] R. Duprat, C. Bourbonnais, Eur. Phys. J. B 21 (2001) 219.

[40] J. C. Nickel, R. Duprat, C. Bourbonnais, N. Dupuis, Phys. Rev. B 73 (2006) 165126.

[41] D. Zanchi, H. J. Schulz, Phys. Rev. B 61 (2000) 13609.

[42] V. J. Emery, Synth. Met. 13 (1986) 21.

[43] L. G. Caron, C. Bourbonnais, Physica 143B (1986) 453. C. Bourbonnais and L. G. Caron, Europhys. Lett. 5 (1988) 209.

[44] 2011. A. Sedeki and D. Bergeron and C. Bourbonnais, to be published.

[45] A. Abanov, A. V. Chubukov, J. Schmalian, Adv. Phys. 52 (2003) 119.

[46] Y. Vilk, A.-M. Tremblay, J. Phys. I (France) (1997). 